\section{O2C.4 PATTERNS OF HEALTH CARE USE FOLLOWING WORK- RELATED INJURY AND ILLNESS IN AUSTRALIAN TRUCK DRIVERS: A LATENT CLASS ANALYSIS}

${ }^{1}$ Ting Xia*, ${ }^{1}$ Ross lles, ${ }^{2}$ Sharon Newnam, ${ }^{3}$ Dan Lubman, ${ }^{1}$ Alex Collie. ${ }^{1}$ Insurance Work and Health Group, Faculty of Medicine, Nursing and Health Sciences, Monash University, St Kilda, Australia; ${ }^{2}$ Accident Research Centre, Clayton, Australia; ${ }^{3}$ Turning Point, Eastern Health, Richmond, Australia

\subsection{6/OEM-2019-EPI.44}

Purpose To identify patterns of health care use in truck drivers with work-related injury or illness and to identify demographic, occupation, injury/condition, claim and geographic factors associated with patterns of care.

Method 13371 accepted workers compensation claims from truck drivers lodged between 2004 and 2013 in the state of Victoria were included. Episodes of health care were categorised according to practitioner type as General Practitioner (GP), Specialist Physician, Mental Health, Surgery, Return to Work, or Physical Therapy. Latent class analysis was used to identify and characterise the distinct profiles of users with different patterns of health service use. Multinomial logistic regression was used to examine the associations between latent class and predictors including demographic, claim and injuryrelated factors.

Results Four profiles of heath service use were identified: (1) Low Service Users (55\% of the sample) were more likely to be younger, have an injury that did not result in time off work and have conditions other than a musculoskeletal injury; (2) High Service Users (10\%) tended to be those who were aged between 45 and 64 years, lived in major cities and had musculoskeletal conditions that resulted in time off work; (3) Physical Therapy Users (25\%) were more likely to be aged between 45 and 64 years, live in major cities and have nontraumatic injuries that resulted in time off work; and (4) GP/ Mental Health Users (10\%) were more likely to be over 24 years of age, from the lowest socio-economic band, be employed by smaller organisations and be claiming benefits for a mental health condition.

Conclusions It is possible to identify distinct patterns of health care use following work-related injury and disease using workers' compensation claims data. Nature of injury/disease, sociodemographic characteristics and geographic proximity to health services affect patterns of care.

\section{C.5 INCREASING COSTS OF OCCUPATIONAL INJURIES IN ASSOCIATION WITH HIGH AMBIENT TEMPERATURES IN ADELAIDE, SOUTH AUSTRALIA, 2000-2014}

Jianjun Xiang*, Alana Hansen, Dino Pisaniello, Peng Bi. School of Public Health, Adelaide University, Adelaide, Australia

\subsection{6/OEM-2019-EPI.45}

Objective To investigate the impact of ambient temperature on compensation costs due to work-related injuries, and to provide an evidence base about the economic benefits of developing workplace heat prevention strategies in a warming climate.

Methods Workers' compensation claims obtained from SafeWork South Australia for 2000-2014 were transformed into daily time series format and merged with meteorological data. The relationship between temperature and compensation costs were estimated using a generalized linear model after controlling for long-term trends, seasonality, and day of week. A piecewise linear spline function was used to account for non-linearity.

Results A total of 464139 workers' compensation claims were reported during the 15 year period in South Australia, resulting in AU\$14.9 billion dollars compensation payment. Overall, it is a reversed V-shaped temperature-cost association. A $1{ }^{\circ} \mathrm{C}$ increase in maximum temperature was associated with a $1.1 \%$ (95\% CI, $0.2 \%-2.0 \%)$ increase in daily injury compensation expenditure below $35.2^{\circ} \mathrm{C}$. Specifically, significant increases of injury costs were observed in males $(1.4 \%$, 95\% CI $0.3 \%-2.5 \%)$, young workers $(3.0 \%$, 95\% CI $1.2 \%-$ $4.9 \%)$, older workers $\geq 65$ years $(2.4 \%$, 95\% CI $0.5 \%-4.4 \%)$, labourers $(2.7 \%, 95 \%$ CI $0.5 \%-4.8 \%)$, machinery operators and drivers $(3.5 \%, 95 \% \mathrm{CI} 1.6 \%-5.3 \%)$ and the following industries: agriculture, forestry, fishing and hunting $(12.3 \%$, 95\% CI 2.2\%-23.3\%); construction (7.8\%, 95\% CI $0.02 \%-$ $16.3 \%)$; and wholesale and retail trade $(2.4 \%$, 95\% CI $0.5 \%$ 4.4\%). Costs for compensating occupational burns and 'skin and subcutaneous tissue diseases' increased by $3.1 \%(95 \% \mathrm{CI}$ $1.2 \%-5.1 \%)$ and $2.7 \% \quad(95 \%$ CI $0.1 \%-5.4 \%)$ respectively, with a $1{ }^{\circ} \mathrm{C}$ increase in maximum temperature.

Conclusion There is a significant association between temperature and work-related injury compensation costs in Adelaide, South Australia for certain subgroups. Heat attributable workers' compensation costs may increase with the predicted rising temperature.

\section{O2C.6 THE ECONOMIC BURDEN OF OCCUPATIONAL INJURIES AND DISEASES IN FIVE EUROPEAN UNION COUNTRIES}

${ }^{1}$ Emile Tompa*, ${ }^{1}$ Amirabbas Mofidi, ${ }^{2}$ Young Jung, ${ }^{3}$ Thijmen van Bree, ${ }^{3}$ Swenneke van den Heuvel, ${ }^{4}$ Frithjof Michaelsen, ${ }^{4}$ Lucas Porsch, ${ }^{3}$ Martijn van Emmerik. ${ }^{1}$ Institute for Work and Health, Toronto, Canada; ${ }^{2}$ MCMaster University, Hamilton, Canada; ${ }^{3}$ TNO, Leiden, The Netherlands; ${ }^{4}$ Valdani, Vicari and Associates, Brussels, Belgium

\subsection{6/OEM-2019-EPI.46}

The objective of this study was to estimate the economic burden of occupational injuries and diseases in five European Union countries for the reference year 2015.

We used a 'bottom up' approach to estimate the economic burden from a societal perspective for Finland, Germany, Italy, The Netherlands, and Poland. Three broad cost categories were considered-direct health care, indirect productivity, and intangible health-related quality of life costs. The methods started with data on newly diagnosed occupational injuries and diseases from calendar year 2015. We considered lifetime costs for cases across all cost categories. Sensitivity analysis was undertaken to assess the impact of key parameters.

Indirect costs represent the largest proportion of total costs (with the exception is Poland), ranging from 66\% for The Netherland to $43 \%$ for Poland. Intangible costs are the second highest, ranging from $49 \%$ for Poland to $21 \%$ for Finland and The Netherlands. Direct costs range from 16\% for Finland to $8 \%$ for Poland.

Average per case costing is highest for The Netherlands ( $€ 75,342)$, followed by Italy ( $€ 58,411)$, German ( $€ 44,919)$, Finland $(€ 43,069)$ and lastly Poland $(€ 38,918)$. Total costs as a percentage of GDP are highest for Poland (10.4\%), followed by Italy (6.7\%), The Netherlands (3.6\%), Germany $(3.3 \%)$ and lastly Finland $(2.7 \%)$. In terms of costs per working population, the value is highest for Italy $(€ 4,956)$, followed by The Netherland (€2,930), Poland (€2,793), Germany $(€ 2,527)$ and lastly Finland $(€ 2,331)$. 